\title{
Motor Neuron Disease and Risk of Cancer: A Population-Based Cohort Study in Denmark
}

This article was published in the following Dove Press journal: Clinical Epidemiology

Trine Toft Sørensen (ID)

Dóra Körmendiné Farkas ${ }^{2}$

Emil Zâl Bjerregaard Riahi $\mathbb{D}^{2}$

Vera Ehrenstein $\mathbb{D D}^{2}$

Victor W Henderson (1D ${ }^{2-4}$

'Department of Public Health, University of Copenhagen, Copenhagen, Denmark;

${ }^{2}$ Department of Clinical Epidemiology, Aarhus University, Aarhus, Denmark; ${ }^{3}$ Department of Epidemiology and Population Health, Stanford University, Stanford, CA, USA; ${ }^{4}$ Department of Neurology and Neurological Sciences, Stanford University, Stanford, CA, USA
Correspondence: Trine Toft Sørensen Department of Public Health, University of Copenhagen, Øster Farimagsgade 5, Copenhagen K 1014, Denmark Email skg654@ku.dk
Background: Some neurogenerative diseases have been linked to a reduced risk of cancer, but the association between motor neuron disease and cancer risk is not well understood. We hypothesized that cancer risk would be lower among those with motor neuron disease and its most common subtype, amyotrophic lateral sclerosis.

Methods: We conducted a population-based cohort study of motor neuron disease and cancer risk using routinely collected data from population-based registries in Denmark. We examined cancer incidence among patients diagnosed with motor neuron disease between January 1980 and December 2013 followed through 2013. Using Danish national cancer rates for the study period, we computed standardized incidence ratios as a measure of relative risks.

Results: In the cohort of 5053 patients with a motor neuron disease, the overall standardized incidence ratio of any cancer was 1.17 (95\% confidence interval [CI], 1.03-1.31); the corresponding standardized incidence ratio for amyotrophic lateral sclerosis was 1.24 (95\% CI, 0.96-1.57). The standardized incidence ratios of any cancer in the cohort with motor neuron disease was 1.52 (95\% CI, 1.22-1.87) for <1 year of follow-up; 0.87 (95\% CI, 0.68-1.09) for years 1-5 of follow-up; and 1.22 (95\% CI, 1.01-1.46) for $>5$ years of followup. Beyond one year of follow-up, patients in the motor neuron disease had elevated standardized incidence ratios for lymphoid leukemia, non-Hodgkin lymphoma, and basal cell skin cancer.

Conclusion: Findings fail to support the hypothesis that motor neuron disease or amyotrophic lateral sclerosis is associated with reduced cancer incidence. An elevated risk of cancer during the first year of follow-up may be attributable to heightened surveillance.

Keywords: amyotrophic lateral sclerosis, cancer, cohort, motor neuron disease, neurodegeneration, risk

\section{Introduction}

Motor neuron disease is a rare, progressive, and usually fatal neurodegenerative condition that causes death of motor neurons in the cerebrum, brainstem, and spinal cord. ${ }^{1}$ Motor neuron disease includes amyotrophic lateral sclerosis and several related disorders. ${ }^{2}$ Other neurodegenerative disorders, including Alzheimer's disease and Parkinson's disease, have been linked to a reduced risk of cancer. ${ }^{3-6}$ It is speculated that reduced trophic support for neurons or programmed cell death, both associated with neurologic disease, may also inhibit cancer-associated cellular proliferation. ${ }^{3}$ Some studies of motor neuron disease reported an inverse association between motor neuron disease and all cancers or site-specific cancers, while others have not, with some evidence suggesting that motor neuron disease may be a paraneoplastic condition. ${ }^{7-13}$ 
We hypothesized that cancer risk would be lower among those with motor neuron disease (all subtypes) and those with amyotrophic lateral sclerosis (the most common form of motor neuron disease). We examined this potential association in a cohort study, using routinely collected data from population-based registries in Denmark.

\section{Materials and Methods}

\section{Setting}

We conducted a nationwide cohort study in Denmark, which has a dynamic population of about 5.8 million people and a tax-funded universal health-care system, with government-maintained nationwide registries. ${ }^{14}$ Upon birth or immigration, every Danish resident is assigned a unique personal identifier, recorded in the Danish Civil Registration System and used in all public records. ${ }^{15}$ The Civil Registration System contains information on sex, date of birth or immigration, and date of death or emigration. Thus, the Civil Registration System enables both exact individual-level linkage of all Danish health registries and complete follow-up of the entire Danish population. ${ }^{15}$

\section{Motor Neuron Disease Cohort}

In the Danish National Patient Registry, we identified a cohort of all patients diagnosed with motor neuron disease (International Classification of Diseases codes in Table 1) seen as inpatients or outpatients in Danish hospitals between 1 January 1980 and 31 December 2013. The Danish National Patient Registry has recorded all inpatient diagnoses and procedures since 1977, and outpatient clinic visits, psychiatric ward visits, and emergency room visits since $1995 .{ }^{16}$ Diagnoses in the Danish National Patient Registry were coded according to the International Classification of Diseases 8th revision from 1977 to 1993 and the 10th revision thereafter. Each hospital discharge or outpatient clinic visit is associated with one primary diagnosis and up to nineteen secondary diagnoses. ${ }^{16}$

\section{Cancer Outcomes}

All members of the cohort were linked through their unique identifiers to the Danish Cancer Registry, which was the source of data on the cancer diagnoses (Table 1). The Danish Cancer Registry has recorded information on all cases of incident primary cancer in Denmark since 1943, with compulsory reporting since 1987. Registration in the Danish Cancer Registry is based on notification of new cancer diagnoses by general practitioners, practicing specialists and hospital
Table I International Classification of Diseases 8th Revision and International Classification of Diseases 10th Revision Used to Identify Study Variables

\begin{tabular}{|c|c|c|}
\hline & ICD-8 & ICD-IO \\
\hline $\begin{array}{l}\text { Amyotrophic } \\
\text { lateral sclerosis } \\
\text { and motor } \\
\text { neuron disease }\end{array}$ & $\begin{array}{l}\text { Motor neuron disease: } \\
348 \text { (amyotrophic } \\
\text { lateral sclerosis } 348.09 \text {, } \\
\text { paralysis bulbaris } \\
\text { progressive } 348.19 \text {, } \\
\text { paralysis spinalis } \\
\text { progressive } 348.20 \text {, } \\
\text { atrophia musculorum } \\
\text { progressiva alia } 348.29 \text {, } \\
\text { morbus neuronis } \\
\text { motorii alius et non } \\
\text { specificatus } 348.99 \text { ) }\end{array}$ & $\begin{array}{l}\text { Motor neuron disease: } \\
\text { GI } 2.2 \\
\text { Amyotrophic lateral } \\
\text { sclerosis: GI2.2G }\end{array}$ \\
\hline Cancer & & $\mathrm{C} 00-\mathrm{C} 96$ \\
\hline $\begin{array}{l}\text { Lung, bronchi and } \\
\text { trachea }\end{array}$ & & C33-C34 \\
\hline $\begin{array}{l}\text { Brain and other } \\
\text { nervous system } \\
\text { cancers }\end{array}$ & & $\begin{array}{l}\text { C70-C72, D32, D42, } \\
\text { D33, D352-D354, } \\
\text { D43, D443- D445 }\end{array}$ \\
\hline $\begin{array}{l}\text { Basal cell } \\
\text { carcinoma }\end{array}$ & & $\begin{array}{l}\text { C44 restricted to } \\
\text { ICD-O-3 morphology: } \\
809\end{array}$ \\
\hline Prostate & & $\mathrm{C} 61$ \\
\hline $\begin{array}{l}\text { Malignant } \\
\text { melanoma }\end{array}$ & & $\mathrm{C} 43$ \\
\hline Breast & & C50 \\
\hline $\begin{array}{l}\text { Non-Hodgkin } \\
\text { lymphoma }\end{array}$ & & C82-C85, C90 \\
\hline Colorectal & & $\mathrm{Cl} 8-\mathrm{C} 19, \mathrm{C} 20$ \\
\hline $\begin{array}{l}\text { Lymphoid } \\
\text { leukemia }\end{array}$ & & C9I \\
\hline Urinary bladder & & $\begin{array}{l}\text { C67, D090, D303, } \\
\text { D4I4 restricted to } \\
\text { ICD-O-3 morphology: } \\
8|2-8| 3\end{array}$ \\
\hline Testicular cancer & & C62 \\
\hline $\begin{array}{l}\text { Salivary gland } \\
\text { cancers }\end{array}$ & & $\mathrm{C} 07-\mathrm{C} 08$ \\
\hline
\end{tabular}

Abbreviations: ICD-8, The International Classification of Diseases 8th Revision; ICD-I0, The International Classification of Diseases I0th Revision.

departments, including departments of pathology and forensic medicine. ${ }^{17}$ Patients with a cancer diagnosis before motor neuron disease diagnosis were excluded. 


\section{Statistical Analyses}

We followed the patients in the motor neuron disease cohort from the date of the first diagnosis of motor neuron disease until date of cancer diagnosis, death, emigration, or December 31, 2013, whichever came first. As a measure of relative risks, we computed standardized incidence ratios as the ratio of observed cancer incidence divided by expected cancer incidence. Expected numbers of cancer cases were calculated based on national incidence rates by age (five-year groups), sex, and calendar period (five-year intervals). Multiplying the number of person-years of observation by the national incidence rates yielded the number of cancer patients expected if patients with motor neuron disease had the same risk of cancer as the general population. ${ }^{18}$ Exact confidence intervals (CIs) were calculated when the observed number was fewer than 10; otherwise, Byar's approximation was used, ${ }^{18}$ assuming that the observed number of cancers followed a Poisson distribution. According to Danish privacy rules, only cells counts larger than four were reported. ${ }^{19}$

We conducted the analyses for any cancer, for common site-specific cancers, and for selected site-specific cancers associated with motor neuron disease in earlier studies. ${ }^{8,9}$ We computed the standardized incidence ratios overall and by follow-up time divided into $<1$ year, years $1-5$, and $>5$ years. We calculated overall standardized incidence ratios, stratified by age groups, sex, and calendar period (before 1994 and 1994 and later, based on the year of transition from the International Classification of Diseases 8th Revision to the International Classification of Diseases 10th Revision).

We conducted a subanalysis for any cancer, restricting the cohort to patients with a specific amyotrophic lateral sclerosis diagnostic code. A specific amyotrophic lateral sclerosis code was available in the International Classification of Diseases 8th revision. However, in the beginning of 10th Revision period (1994 to approx. 2000), the specific code for amyotrophic lateral sclerosis was not widely used. The proportion of patients with a specific ICD-10 code for amyotrophic lateral sclerosis was 2\% among patients diagnosed in 1994-2000 and 16\% among patients diagnosed in 2001-2013.

\section{Ethical Aspects}

This study was approved by the Danish Data Protection Agency (record number AU 456). According to Danish law, studies based entirely on registry data do not require patient consent or approval from an ethics review board.

\section{Results}

We identified 5053 patients (2258 women and 2795 men) with a first-time diagnosis of motor neuron disease between 1980 and 2013. The median age at diagnosis was 65.8 years (interquartile range [IQR]: 56.1-73.5) and the median follow-up was 1.3 years. In this cohort, 1542 patients had a specific diagnostic code for amyotrophic lateral sclerosis (median age at diagnosis 67.3 years, IQR: 59.2-74.2; median follow-up 0.8 years). Distribution of all other diagnostic codes is presented in the Supplemental Table.

The standardized incidence ratio of any cancer, associated with motor neuron disease, was 1.17 (95\% CI, 1.03-1.31): 1.31 (95\% CI, 1.08-1.58) among women and 1.08 (95\% CI, 0.92-1.26) among men for the entire 33-year follow-up period. The standardized incidence ratios for the period 1980 to 1993 were 1.28 (95\% CI, 1.06-1.54) and 1.09 (95\% CI, 0.93-1.27) thereafter. Patients diagnosed with motor neuron disease before the age of 60 years had a higher standardized incidence ratio for any cancer $(1.32$ [95\% CI, 1.08-1.60]) than older patients (1.09 [95\% CI, $0.94-1.26])$.

Patients with motor neuron disease were more likely to be diagnosed with cancer than individuals of same sex and similar age in the general population during the first year of follow-up (Table 2). During the first year of follow-up, 89 cancers were identified in patients with motor neuron disease, yielding a standardized incidence ratio for cancer of 1.52 (95\% CI, 1.22-1.87). For follow-up beyond the first year, the standardized incidence ratio for any cancer in the cohort with motor neuron disease was 0.87 (95\% CI, 0.68-1.09) for years $1-5$ and 1.22 (95\% CI, 1.01-1.46) for $>5$ years of follow-up. During the first year of follow-up, motor neuron disease was associated with several cancer sites, but the risk estimates were imprecise (Table 2). For cancer of the brain and other nervous system tumors, the standardized incidence ratio was 11.54 (95\% CI, 6.59-18.8). For malignant melanoma, the standardized incidence ratio was 3.76 (95\% CI, 1.22-8.76), and for lung cancer, the standardized incidence ratio was 2.30 (95\% CI, 1.32-3.74).

Beyond one year of follow-up, elevated standardized incidence ratios were observed for lymphoid leukemia (2.91 [95\% CI, 0.60-8.50]) in years $1-5$, and $(2.64$ [95\% CI, 0.54-7.70]) for $>5$ years; non-Hodgkin lymphoma $(2.13$ [95\% CI, 0.78 4.65] and 1.92 [95\% CI, 0.71-4.19]), and basal cell skin cancer (1.38 [95\% CI, 0.86-2.08] and 1.73 [95\% CI, 1.19-2.45]). The standardized incidence ratios for lung cancer were 0.39 (95\% 
Table 2 Standardized Incidence Ratios for Selected Cancers Among Patients with Motor Neuron Diseases

\begin{tabular}{|c|c|c|c|c|c|c|c|c|c|}
\hline \multirow[t]{2}{*}{ Cancer site } & \multicolumn{3}{|c|}{$<$ I year of follow-up } & \multicolumn{3}{|c|}{ I-5 years of follow-up } & \multicolumn{3}{|c|}{$\geq 5$ years of follow-up } \\
\hline & Observed & Expected & SIR (95\% CI) & Observed & Expected & SIR (95\% CI) & Observed & Expected & SIR (95\% CI) \\
\hline All & 89 & 58.7 & $1.52(1.22-1.87)$ & 76 & 87.5 & $0.87(0.68-1.09)$ & 118 & 96.7 & $1.22(1.01-1.46)$ \\
\hline Colorectal & $<5$ & NR & $0.45(0.09-1.32)$ & 7 & 9.7 & $0.72(0.29-1.49)$ & 7 & 10.6 & $0.66(0.27-1.37)$ \\
\hline $\begin{array}{l}\text { Lung, bronchi and } \\
\text { trachea }^{\mathrm{a}}\end{array}$ & 16 & 6.9 & $2.30(1.32-3.74)$ & $<5$ & NR & $0.39(0.11-1.00)$ & 13 & 10.9 & $1.19(0.63-2.04)$ \\
\hline Malignant melanoma ${ }^{b}$ & 5 & 1.3 & $3.76(1.22-8.76)$ & $<5$ & NR & $0.47(0.01-2.64)$ & $<5$ & NR & $0.81(0.10-2.93)$ \\
\hline Breast & $<5$ & NR & $0.39(0.05-1.42)$ & 8 & 6.7 & $1.19(0.51-2.34)$ & 8 & 6.5 & $1.24(0.53-2.44)$ \\
\hline Prostate $^{\mathrm{a}}$ & 5 & 6.0 & $0.83(0.27-1.94)$ & $<5$ & NR & $0.41(0.11-1.06)$ & 13 & 11.7 & I.II (0.59-1.90) \\
\hline Testicle $^{b}$ & 0 & 0.1 & - & $<5$ & NR & $3.64(0.09-20.29)$ & $<5$ & NR & $5.39(0.65-19.46)$ \\
\hline Urinary bladder & $<5$ & NR & $0.61(0.07-2.22)$ & $<5$ & NR & $0.20(0.01-1.12)$ & 6 & 5.5 & $1.08(0.40-2.36)$ \\
\hline $\begin{array}{l}\text { Brain and other } \\
\text { nervous system }\end{array}$ & 16 & 1.4 & II.54 (6.59-18.75) & 0 & 2.1 & - & 6 & 2.4 & $2.54(0.93-5.54)$ \\
\hline $\begin{array}{l}\text { Non-Hodgkin } \\
\text { malignant lymphoma }\end{array}$ & $<5$ & NR & $1.60(0.33-4.68)$ & 6 & 2.8 & $2.13(0.78-4.65)$ & 6 & 3.1 & $1.92(0.71-4.19)$ \\
\hline Lymphoid leukemia & $<5$ & NR & $2.89(0.35-10.45)$ & $<5$ & NR & $2.91(0.60-8.50)$ & $<5$ & NR & $2.64(0.54-7.70)$ \\
\hline Basal cell skin cancer & 9 & 10.4 & $0.86(0.39-1.64)$ & 22 & 16.0 & $1.38(0.86-2.08)$ & 32 & 18.4 & $1.73(1.19-2.45)$ \\
\hline $\begin{array}{l}\text { Other cancer sites } \\
\text { combined }\end{array}$ & 26 & 14.9 & $1.75(1.14-2.56)$ & 19 & 21.8 & $0.87(0.53-1.36)$ & 20 & 23.4 & $0.85(0.52-1.32)$ \\
\hline
\end{tabular}

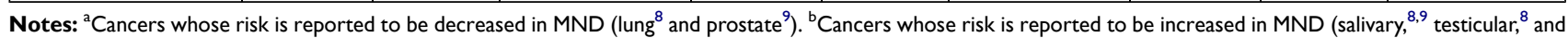
melanoma ${ }^{8}$ ). There were no observed cases of salivary gland cancer during the follow-up.

Abbreviations: $\mathrm{Cl}$, confidence interval; $\mathrm{NR}$, not reportable (to prevent back-calculation from SIR to comply with the Danish privacy regulations ${ }^{19}$ ); MND, motor neuron disease; SIR, standardized incidence ratio.

CI, 0.11-1.00) for years $1-5$ and $1.19(95 \%$ CI, 0.63-2.04) for $>5$ years of follow-up; the respective estimates for colorectal cancer were 0.72 (95\% CI, 0.29-1.49) and 0.66 (95\% CI, $0.27-1.37)$. There were no observed cases of salivary gland cancer. Many cancer sites had fewer than 5 observed cases and the associated estimates are therefore imprecise.

For the 1542 patients with a specific amyotrophic lateral sclerosis diagnostic code, the standardized incidence ratio for any cancer was $1.24(95 \% \mathrm{CI}, 0.96-1.57)$ for the entire 33-year follow-up period, 1.82 (95\% CI, 1.21-2.64) for the first year and 1.00 (95\% CI, 0.71-1.37) for the subsequent years.

\section{Discussion}

In this nationwide population-based cohort study, we found no evidence of a reduced risk of cancer in patients with motor neuron disease. Instead, during the first year following a diagnosis of motor neuron disease, patients had about $52 \%$ elevated risk of any cancer compared with the general population of the same sex and similar age. After one year, risks of any cancer were similar in the patients with motor neuron disease and in the general population. Results for patients with a specific diagnosis of amyotrophic lateral sclerosis were broadly consistent with the overall results.

Several studies have shown reductions in risk of cancer in neurodegenerative disorders like Alzheimer's disease and Parkinson's disease. ${ }^{3-5}$ Speculatively, inverse associations could reflect opposing physiological and pathological processes related to neuronal cell death in neurodegenerative disorders and to uncontrolled cell proliferation in malignancy. ${ }^{3}$ An alternative explanation for the observed association is that clinicians may be less likely to screen for certain cancers or reluctant to pursue cancer diagnoses aggressively in patients with serious neurologic morbidity. Our finding of no overall cancer risk reduction in patients with motor neuron 
disease fails to support the inverse association hypothesis, at least as it pertains to this particular neurodegenerative disorder.

Our findings extend earlier evidence on the relation between motor neuron disease and cancer. ${ }^{3,7-9,14,20}$ Reported associations in some studies are based on incident motor neuron disease after a cancer diagnosis rather than the other way around, as in our study. Bi-directional inverse associations could imply causal mechanisms operating to increase cancer risk in neurodegenerative disease and to increase neurodegeneration in cancer. The two hypotheses, although related, are not one and the same and might differ for motor neuron disease as compared with Alzheimer's disease or Parkinson's disease.

A British population-based record linkage study showed no overall cancer risk increase among 838 motor neuron disease patients compared with a control cohort of people with other specified medical disorders (rate ratio 0.98; 95\% CI, 0.75-1.26). Data from the Surveillance, Epidemiology and End Results Program in the US, which included 1216 motor neuron disease deaths, did not show an association between motor neuron disease mortality among one-year cancer survivors (standardized mortality ratio $1.00 ; 95 \% \mathrm{CI}, 0.95-1.06){ }^{20}$ In contrast, a Swedish-nested case-control study of amyotrophic lateral sclerosis patients and general-population controls reported a lower risk of cancer diagnosis in 4926 amyotrophic lateral sclerosis patients (incidence rate ratio 0.84 ; 95\% CI, 0.69-1.02). ${ }^{7}$ The risk reduction was seen primarily two or more years after amyotrophic lateral sclerosis diagnosis (incidence rate ratio 0.64; 95\% CI, 0.45-0.88). However, the Swedish investigators also found no association between a history of cancer and subsequent risk of amyotrophic lateral sclerosis (odds ratio: 1.00; 95\% CI, 0.91-1.10), concluding that there was no overall evidence for correlation between cancer and amyotrophic lateral sclerosis. ${ }^{7}$ A US study using a Utah genealogy database of 1081 patients with death certificate diagnoses of motor neuron disease reported a decreased lifetime risk of cancer in these patients (hazard ratio: $0.80 ; 95 \% \mathrm{CI}, 0.66-0.96$ ). ${ }^{8}$

With respect to site-specific cancers, we found increased risks for brain and central nervous system tumors, melanoma, and lung cancer during the first year after a motor neuron disease diagnosis and increased risks for lymphoid leukemia, non-Hodgkin lymphoma, and basal cell cancer after one year. In contrast, lung cancer risk was reduced in the Utah study (hazard ratio: 0.23; 95\% CI, 0.05-0.63), where hazards were also increased for salivary and testicular cancers. ${ }^{8}$ There were no cases of these cancers in our motor neuron disease cohort.

The elevated risks of cancer during the first year of follow-up in this study are likely attributable to heightened surveillance related to diagnostic procedures (CT or MRI scan of the head and cervical spine), the clinical examination (visual inspection of unrobed patients for muscle atrophy and fasciculations; strength testing of individual muscle groups), and the clinical assessment of patients with weakened respiratory muscles and impaired swallowing, which might predispose to pulmonary infection (chest X-rays). In the motor neuron disease cohort, the early increased risk of lung cancer was followed by a slight, possibly compensatory, deficit in the standardized incidence ratio after one year. The increased risk of basal cell cancer beyond one year of follow-up may also be explained by more intense visual inspection of patients during the motor examination. More frequent hospital contact with heightened surveillance may also account for increased numbers of some hematological cancers (lymphoid leukemia, non-Hodgkin lymphoma).

In a British study, more cases than expected of amyotrophic lateral sclerosis were associated with autoimmune disorders, such as myasthenia gravis, myxedema, polymyositis and other autoimmune diseases. ${ }^{21}$ The risk of non-Hodgkin lymphoma is elevated in patients with autoimmune and chronic inflammatory diseases, ${ }^{21-23}$ and future research might examine associations based on immunological mechanisms. We were unable to corroborate the finding by Gibson and colleagues of a reduced risk of several site-specific cancers among patients with motor neuron diseases. ${ }^{8}$ Moreover, in contrast to previous studies, ${ }^{8,9}$ we found elevated risks of basal cell cancer, lymphoid leukemia and non-Hodgkin lymphoma, although the number of cases was small.

\section{Strengths and Limitations}

Our study was based on data from nationwide populationbased registries that allowed for complete long-term followup. Use of routinely collected health data, accrued in a hypothesis-independent fashion reduces the risk of information bias. ${ }^{14}$ The Danish national health service with universal access to health care reduces referral and diagnostic biases. A validation study showed that $85 \%$ of patients coded with motor neuron disease in the Danish National Patient Registry had amyotrophic lateral sclerosis (65\%) or another motor neuron disease (20\%), while $8 \%$ had Parkinson's disease and $4 \%$ were without a final diagnosis. ${ }^{24}$ With the 
exception of non-melanoma skin cancer, data in the Danish Cancer Registry are nearly complete and valid due to compulsory reporting, and most tumors since 2009 have been histologically confirmed. ${ }^{25}$ Despite the comparatively large size of the motor neuron disease cohort, estimates for some site-specific cancers were imprecise, due to a small number of events. Some patients with amyotrophic lateral sclerosis diagnosed from 1994 to 2000 were not included in the specific amyotrophic lateral sclerosis analysis, because specific International Classification of Disease coding was not available during this period.

Motor neuron disease may be etiologically heterogeneous. It is linked to genetic mutations and possibly to physical trauma, toxins, and other exposures. ${ }^{26}$ It is thus possible that associations with cancer in general or with specific cancers might differ according to disease etiology. In the Danish registries, amyotrophic lateral sclerosis was the most common diagnostic phenotype, and cancer risk in this subgroup is similar to that in the larger motor neuron disease cohort. However, amyotrophic lateral sclerosis itself has different causes, and we cannot rule out subgroup differences.

\section{Conclusions}

Our large population-based study showed no reduced risk of cancer in patients with motor neuron disease. Our findings thus do not support the hypothesis of an inverse association between neurodegeneration and cancer. Lack of an inverse association has been previously reported for Parkinson's disease, ${ }^{6}$ although evidence is conflicting, and a putative inverse association may be specific for Alzheimer's disease. Alternatively, apparent risk reduction in Alzheimer's disease might also reflect bias linked to reduced cancer surveillance. We were unable to corroborate previous reports of reduced risk of several site-specific cancers among patients with motor neuron disease. Moreover, we found elevated risks of basal cell cancer, lymphoid leukemia and non-Hodgkin lymphoma in follow-up after one year, although numbers of cases were small and the associated estimates imprecise.

\section{Acknowledgment}

The authors thank Professor Henrik Toft Sørensen for his valuable comments, which helped improve the manuscript.

\section{Funding}

This study was supported by a grant from the Lundbeckfonden (Grant No. R248-2017-521). VWH was supported by the National Institutes of Health (Grant Nos. P50AG047366 and P30AG066515).

\section{Disclosure}

The authors report no conflicts of interest in this work.

\section{References}

1. Brown RH, Al-Chalabi A. Amyotrophic lateral sclerosis. $N$ Engl $J$ Med. 2017;377(2):162-172. doi:10.1056/NEJMra1603471

2. Feigin VL, Abajobir AA, Abate KH, et al. Global, regional, and national burden of neurological disorders during 1990-2015: a systematic analysis for the Global Burden of Disease Study 2015. Lancet Neurol. 2017;16(11):877-897. doi:10.1016/S1474-4422(17)30299-5

3. Driver JA. Inverse association between cancer and neurodegenerative disease: review of the epidemiologic and biological evidence. Biogerontology. 2014;15(6):547-557. doi:10.1007/s10522-014-9523-2

4. Ganguli M. A reduced risk of Alzheimer's disease in those who survive cancer. BMJ. 2012;344:e1662. doi:10.1136/bmj.e1662

5. Catala-Lopez F, Suarez-Pinilla M, Suarez-Pinilla P, et al. Inverse and direct cancer comorbidity in people with central nervous system disorders: a meta-analysis of cancer incidence in 577,013 participants of 50 observational studies. Psychother Psychosom. 2014;83 (2):89-105. doi:10.1159/000356498

6. Ording AG, Veres K, Horvath-Puho E, et al. Alzheimer's and Parkinson's diseases and the risk of cancer: a cohort study. J Alzheimers Dis. 2019;72(4):1269-1277.

7. Fang F, Al-Chalabi A, Ronnevi LO, et al. Amyotrophic lateral sclerosis and cancer: a register-based study in Sweden. Amyotroph Lateral Scler Frontotemporal Degener. 2013;14(5-6):362-368. doi:10.3109/21678421.2013.775309

8. Gibson SB, Abbott D, Farnham JM, et al. Population-based risks for cancer in patients with ALS. Neurology. 2016;87(3):289-294. doi:10.1212/WNL.0000000000002757

9. Fois AF, Wotton CJ, Yeates D, Turner MR, Goldacre MJ. Cancer in patients with motor neuron disease, multiple sclerosis and Parkinson's disease: record linkage studies. J Neurol Neurosurg Psychiatry. 2010;81(2):215-221. doi:10.1136/jnnp.2009.175463

10. Ording AG, Horvath-Puho E, Veres K, et al. Cancer and risk of Alzheimer's disease: small association in a nationwide cohort study. Alzheimers Dement. 2020;16(7):953-964. doi:10.1002/alz.12090

11. Akan O, Baysal-Kirac L. Amyotrophic lateral sclerosis with coexisting cancer: a single-center study. Acta Neurol Belg. 2020. doi:10.1007/s13760-020-01337-y

12. Verschueren A, Gallard J, Boucraut J, Honnorat J, Pouget J, Attarian S. Paraneoplastic subacute lower motor neuron syndrome associated with solid cancer. $J$ Neurol Sci. 2015;358(1-2):413-416. doi:10.1016/j.jns.2015.08.014

13. Mele N, Berzero G, Maisonobe T, et al. Motor neuron disease of paraneoplastic origin: a rare but treatable condition. $J$ Neurol. 2018;265(7):1590-1599. doi:10.1007/s00415-018-8881-0

14. Schmidt M, Schmidt SAJ, Adelborg K, et al. The Danish health care system and epidemiological research: from health care contacts to database records. Clin Epidemiol. 2019;11:563-591. doi:10.2147/ CLEP.S179083

15. Schmidt M, Pedersen L, Sorensen HT. The Danish Civil Registration System as a tool in epidemiology. Eur J Epidemiol. 2014;29 (8):541-549. doi:10.1007/s10654-014-9930-3

16. Schmidt M, Schmidt SA, Sandegaard JL, Ehrenstein V, Pedersen L, Sorensen HT. The Danish National Patient Registry: a review of content, data quality, and research potential. Clin Epidemiol. 2015;7:449-490. doi:10.2147/CLEP.S91125

17. Gjerstorff ML. The Danish Cancer Registry. Scand J Public Health. 2011;39(7Suppl):42-45. doi:10.1177/1403494810393562 
18. Breslow NE, Day NE. Statistical methods in cancer research. Volume IIThe design and analysis of cohort studies. IARC Sci Publ. 1987;82:1-406.

19. Data Privacy Policy. Available from: https://www.dst.dk/ext/formid/ datafortrolighed-pdf. Accessed September 22, 2020.

20. Freedman DM, Curtis RE, Daugherty SE, Goedert JJ, Kuncl RW, Tucker MA. The association between cancer and amyotrophic lateral sclerosis. Cancer Causes Control. 2013;24(1):55-60. doi:10.1007/ s10552-012-0089-5

21. Turner MR, Goldacre R, Ramagopalan S, Talbot K, Goldacre MJ. Autoimmune disease preceding amyotrophic lateral sclerosis: an epidemiologic study. Neurology. 2013;81(14):1222-1225. doi:10.1212/ WNL.0b013e3182a6cc13

22. Hemminki K, Huang W, Sundquist J, Sundquist K, Ji J. Autoimmune diseases and hematological malignancies: exploring the underlying mechanisms from epidemiological evidence. Semin Cancer Biol. 2020;64:114-121. doi:10.1016/j.semcancer.2019.06.005

23. Ansell P, Simpson J, Lightfoot T, et al. Non-Hodgkin lymphoma and autoimmunity: does gender matter? Int $J$ Cancer. 2011;129 (2):460-466. doi:10.1002/ijc. 25680
24. Sorensen HT, Riis AH, Lash TL, Pedersen L. Statin use and risk of amyotrophic lateral sclerosis and other motor neuron disorders. Circ Cardiovasc Qual Outcomes. 2010;3(4):413-417. doi:10.1161/ CIRCOUTCOMES.110.936278

25. Coleman MP, Forman D, Bryant $\mathrm{H}$, et al. Cancer survival in Australia, Canada, Denmark, Norway, Sweden, and the UK, 1995-2007 (the International Cancer Benchmarking Partnership): an analysis of population-based cancer registry data. Lancet. 2011;377 (9760):127-138. doi:10.1016/S0140-6736(10)62231-3

26. Wang M-D, Little J, Gomes J, Cashman NR, Krewski D. Identification of risk factors associated with onset and progression of amyotrophic lateral sclerosis using systematic review and meta-analysis. Neurotoxicology. 2017;61:101-130. doi:10.1016/j. neuro.2016.06.015

\section{Publish your work in this journal}

Clinical Epidemiology is an international, peer-reviewed, open access, online journal focusing on disease and drug epidemiology, identification of risk factors and screening procedures to develop optimal preventative initiatives and programs. Specific topics include: diagnosis, prognosis, treatment, screening, prevention, risk factor modification,

Submit your manuscript here: https://www.dovepress.com/clinical-epidemiology-journal systematic reviews, risk \& safety of medical interventions, epidemiology \& biostatistical methods, and evaluation of guidelines, translational medicine, health policies \& economic evaluations. The manuscript management system is completely online and includes a very quick and fair peer-review system, which is all easy to use. 